\title{
PACE: Simple Multi-hop Scheduling for Single-radio 802.11-based Stub Wireless Mesh Networks
}

\author{
Filipe Ribeiro, Rui Campos, David Rua, Carlos Pinho, José Ruela \\ INESC TEC (formerly INESC Porto), Universidade do Porto \\ Rua Dr. Roberto Frias, 378 \\ Porto, Portugal \\ Email: filipe.a.ribeiro@inescporto.pt
}

\begin{abstract}
IEEE 802.11-based Stub Wireless Mesh Networks (WMNs) are a cost-effective and flexible solution to extend wired network infrastructures. Yet, they suffer from two major problems: inefficiency and unfairness. A number of approaches have been proposed to tackle these problems, but they are too restrictive, highly complex, or require time synchronization and modifications to the IEEE 802.11 MAC.

PACE is a simple multi-hop scheduling mechanism for Stub WMNs overlaid on the IEEE 802.11 MAC that jointly addresses the inefficiency and unfairness problems. It limits transmissions to a single mesh node at each time and ensures that each node has the opportunity to transmit a packet in each network-wide transmission round. Simulation results demonstrate that PACE can achieve optimal network capacity utilization and greatly outperforms state of the art CSMA/CA-based solutions as far as goodput, delay, and fairness are concerned.
\end{abstract}

Keywords: IEEE 802.11, Single-radio, Wireless Mesh Network, Scheduling, Multi-hop, Fairness

\section{INTRODUCTION}

The increasing number of IEEE 802.11 devices is raising the demand for IEEE 802.11 accesses. Since IEEE 802.11 has limited radio range, covering large geographical areas can only be achieved by installing multiple Access Points (APs) that need to be directly connected to the wired backhaul infrastructure. This may require the installation of a large number of APs and wires, which introduces complexity and may imply high financial costs and considerable technical manpower to deploy the network. The need to connect each AP to the wired infrastructure reduces flexibility, as well.

Wireless Mesh Networks (WMNs) are considered a flexible and cost-effective solution to overcome those problems. In WMNs, nodes cooperate with each other to wirelessly forward data between sources and destinations. WMNs may be defined in different ways. Herein, an $802.11 \mathrm{WMN}$ is assumed to be a wireless network used to extend the coverage of a wired infrastructure. Such a Stub WMN, as will be called in this paper, consists of static Mesh Access Points (MAPs) that perform multi-hop bidirectional forwarding between the wired infrastructure and the wireless terminals attaching to MAPs. The latter are assumed to have up to two Network Interface Cards (NICs), one used for connecting to the WMN and the other that can be used to serve wireless terminals. The reference scenario for an 802.11 Stub WMN is illustrated in Fig. 1.

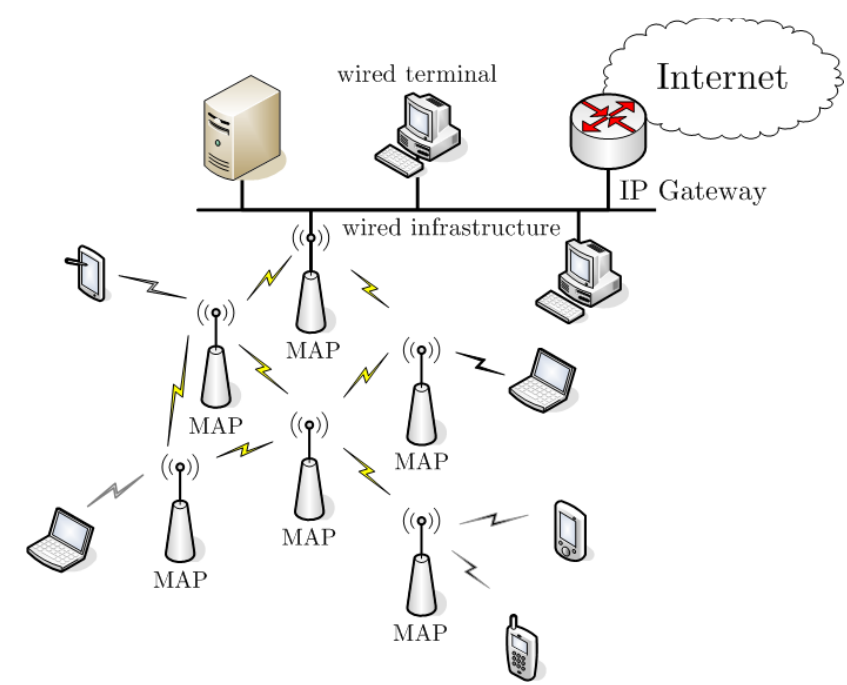

Figure 1. Stub Wireless Mesh Networking Scenario.

Existing WMNs suffer from two major problems: inefficiency and unfairness. Inefficiency comes from using the Carrier Sense Multiple Access with Collision Avoidance (CSMA/CA) medium access mechanism, which was designed for single-hop networks. CSMA/CA cannot avoid frame collisions effectively due to hidden nodes induced by the multihop topology. Frame collisions lead to retransmissions and higher number of frames sent out per packet to be transmitted, with low network transmission efficiency and, consequently, reduced network throughput and high network delays [1]. The wireless medium is unfairly shared among nodes due to the multi-hop nature of the network; nodes closer to the wired infrastructure take control of the medium and cause other nodes to starve, even for small Stub WMNs [1].

A number of approaches have been proposed to tackle these issues. IEEE 802.11 has built-in mechanisms to address the hidden node problem and enable fair and coordinated medium access control, but performance degradation occurs in multihop topologies [2]. Enhancements at the physical and MAC layers [3, 4] are important, but solving the most challenging problems in WMNs requires additional mechanisms that may be implemented at higher layers. However, the proposed solutions are either too restrictive (only solving specific problems or addressing particular scenarios) or highly complex and introduce too much overhead, or may even require time synchronisation and modifications to the MAC layer. Thus, a simple solution compatible with the 802.11 MAC and jointly addressing the problems mentioned above is lacking. 
Herein, we propose PACE, a scheduling mechanism for Stub WMNs overlaid on the IEEE 802.11 MAC and based on WiFIX, a simple and efficient tree-based routing solution for Stub WMNs proposed in $[1,5]$. In each MAP, WiFIX runs on top of the IEEE 802.11 wireless card and processes all incoming/outgoing data packets. PACE leverages WiFIX in order to control data packet transmissions over the air interface. It jointly addresses the inefficiency and unfairness problems by limiting transmissions to a single mesh node at each time and ensuring that each node has the opportunity to transmit a packet in each network-wide transmission round.

Our contribution is two-fold. Firstly, we propose PACE, a collision-free asynchronous time division multi-hop medium access mechanism, which enables efficient and fair single-radio Stub WMNs using the 802.11 legacy MAC, without: (1) any predetermined resource reservation and allocation mechanism, (2) enforced temporal synchronisation among MAPs, (3) the exchange of state information between MAPs (e.g., queue sizes), (4) a fixed data packet size, and (5) any congestion control needs. Secondly, we demonstrate that it is possible to define a simple, optimal scheduling mechanism for single-radio Stub WMNs without modifying the 802.11 legacy MAC and introducing the complexity found in state of the art solutions.

The rest of the paper is structured as follows. Section II reviews the state of the art and Section III gives an overview of the WiFIX solution. Section IV describes the PACE scheduling mechanism and the corresponding WiFIX modifications. Section V refers to WiFIX and PACE implementations under ns-3 and Section VI evaluates PACE against CSMA/CA, based on ns-3 simulations results. Section VII concludes the paper and points out to future plans.

\section{STATE OF THE ART}

In CSMA/CA networks, the simultaneous transmission of nodes that are outside each others carrier sensing range may cause collisions. To mitigate this hidden node $(\mathrm{HN})$ problem, an RTS/CTS handshake mechanism is specified in IEEE 802.11. Nodes that hear an RTS or CTS are blocked (not allowed to transmit) even if no interference would result; this is the root cause of the exposed node (EN) problem that reduces the level of spatial reuse. The trade-off between HN and EN, both causing performance degradation, depends on relative values of transmission, carrier sensing and interference ranges.

These problems are aggravated in 802.11-based WMNs, due to multi-hop forwarding. The network capacity of a wireless link is determined by spatial contention among neighbour links and depends on the distribution of nodes, among other factors. Intra-flow interference due to competition among nodes that forward packets of the same flow over multiple hops further contributes to reducing the overall throughput. As discussed in [6], besides inefficiency, there is also unfairness due to $\mathrm{HN}$ and low scalability due to EN, the latter meaning that the sum of 1-hop throughputs is nonscalable.

Solutions to these problems are approached from different perspectives and may involve a number of related issues, such as spatial reuse, fairness, scheduling and congestion control. Improvements at the PHY and/or MAC layers proposed by many authors are somehow related with the RTS/CTS scheme and may require modifications to the standard.
The effectiveness of RTS/CTS in reducing interference in multi-hop networks due to $\mathrm{HN}$ is analysed in [7], considering the interference range (as a function of the transmitter-receiver distance) and different possible settings for the carrier sensing range. To overcome limitations of the mechanism the authors propose a conservative scheme in which CTS replies are only generated in case the received power of an RTS packet exceeds a certain threshold.

Another issue is the false blocking problem addressed in [2]. It is also a side effect of RTS/CTS handshake and may seriously degrade performance in multi-hop networks. This false blocking not only reduces throughput but may also induce a deadlock-like situation due to a cascading effect. The authors propose an RTS validation scheme based on assessing the state of the channel at the time the expected data packet should start transmission following the corresponding RTS request.

In [8] the authors extend the analysis of [7] considering other relevant scenarios that lead to $\mathrm{HN}$, including a capture effect that may unnecessarily block a receiver, as well as the fact that ACK frames may corrupt or be corrupted by data frames. They derive two requirements for a so-called HN-Free Design: a minimum physical carrier sensing range and use of restart mode by receivers, which must lock on a stronger signal while in the course of receiving a weaker one. This mode solves the capture effect and helps reducing EN.

Further improvements are proposed in [6], which also tackle the EN problem. Formal definitions of EN and $\mathrm{HN}$ are given, based on the analysis of physical and protocol constraints, which are translated into interference and both transmitter and receiver carrier sensing graphs. Relaxing some constraints by disabling or modifying specific 802.11 mechanisms is proposed to solve the $\mathrm{EN}$ and $\mathrm{HN}$ problems and thus achieve scalability and fairness. The solutions are mainly evaluated in the case of multiple independent access points and thus it is not clear how they would behave in WMNs where neighbour nodes have to cooperate to forward transit traffic across multiple hops, while injecting their own traffic.

To complete this topic, it is worth mentioning that techniques to improve spatial reuse, either temporal (window adaptation) or spatial (transmission power control, use of directional antennas, tuning of carrier sensing threshold and rate adaptation) are analysed in [3], while an updated survey of MAC layer solutions to the HN problem is presented in [4].

Although spatial reuse may be improved by techniques that mitigate the EN problem in the close neighbourhood of a transmitting node, solving the general problem requires a global view of WMNs and additional mechanisms that allow simultaneous transmissions on non conflicting links, possibly achieving other goals, such as fairness. Interference models are necessary for this purpose.

A 2-hop interference model is usually adopted in 802.11 WMNs [9, 10]; it means that nodes within one hop of the sender or the receiver of an active link cannot participate in a communication. Link contention graphs are sometimes used to describe the spatial contention relationship among links; only one link can be transmitting at any time in a group of mutually contending links (called a clique).

One simple case addressed by many authors is the chain topology that, nevertheless, allows capturing the effect of inter and intra-flow interference. Factors that determine chain 
behaviour are analysed in [11] and simulation results are presented in $[12,13]$. Flows with long paths are penalised and may even starve due to multiple contentions and packet drops; besides unfairness, overall throughput decreases due to such wastage of resources, which is confirmed in the present paper.

WMNs differ from wired ones due to interference and the time varying nature of wireless channels, which justifies revisiting the classical fairness criteria. A reference model is proposed in [13] with four main goals: 1) fairness among nodeaggregated flows; 2) maximal spatial reuse; 3) elimination of spatial bias (favouring max-min fairness); 4) time as the basic resource to fairly share.

Scheduling algorithms may be designed with fairness in mind. Examples are given in [14], together with a comparison by degree of fairness, metrics and mechanisms.

When scheduling is treated as an optimisation problem the goal is maximising a utility function, e.g. throughput or a function of relevant parameters, under interference constraints $[10,15,16]$. A schedule is as a sequence of fixed length time slots; each possible transmission is assigned a slot in a non colliding way. The optimisation problem must be solved for each time slot and repeated when the topology or traffic loads change; this is not practical in rather dynamic conditions, but may be suitable for long term traffic engineering purposes.

Scheduled link activation schemes (as opposed to random access methods) are studied in [15]. In this seminal paper, it is proved that for multiple traffic classes, a maximum throughput policy called Maximum Weight Scheduling (MWS) tends to equalize the queue lengths of the same class in different nodes, giving priority to links and classes for which this difference is larger. This result has been exploited in cross-layer schemes that implement backpressure congestion control; differential backlog schemes based on the queue sizes on neighbour nodes are described in $[17,18]$.

Since the general problem is typically NP-hard [15, 19], suboptimal solutions must be found. Cross-layer mechanisms, such as Maximal Scheduling (MS) and variants [16], have been studied as practical alternatives to WMS.

We may conclude that the reviewed solutions are either too restrictive, only solving specific problems or addressing particular scenarios, or highly complex, introducing too much processing and control overhead, or unable to fast adapt to dynamic changes.

\section{WIFIX}

WiFIX [1, 5] is a simple and efficient tree-based routing solution for Stub WMNs overlaid on the 802.11 MAC. WiFIX solves the path auto-configuration problem by configuring an active tree topology rooted at the node directly connected to the wired infrastructure (master MAP). For that purpose, it defines a single-message protocol that enables the self-organisation of the Stub WMN, and reuses concepts such as $802.1 \mathrm{D}$ bridges and their simple learning mechanism for frame forwarding. In order to support multi-hop forwarding within a Stub WMN based on legacy IEEE 802.1D bridges, it defines a new encapsulation method, called Ethernet-over-802.11 (Eo11), which enables the creation of virtual links (Eo11 tunnels) between neighbour MAPs. The Active Topology Creation and Maintenance (ATCM) mechanism is used to create the virtual links; together they form the active tree topology rooted at the master MAP.

ATCM works as follows. The master MAP periodically sends a Topology Refresh (TR) message, which is forwarded by all other MAPs, after changing a few parameters (number of hops to the master, parent address, and original address of the frame). Each MAP selects a parent node in the tree rooted at the master MAP. The TR message is employed to both announce the master MAP and notify a node that it has been selected as parent in the tree. IEEE 802.1D bridges are used for packet forwarding on top of the active tree topology; they see the virtual links as common Ethernet links.

WiFIX runs on top of the IEEE 802.11 card and processes all incoming/outgoing data packets, as shown in Fig. 2. This enables the design of a scheduling mechanism on top of the IEEE 802.11 MAC, as described in Section IV.

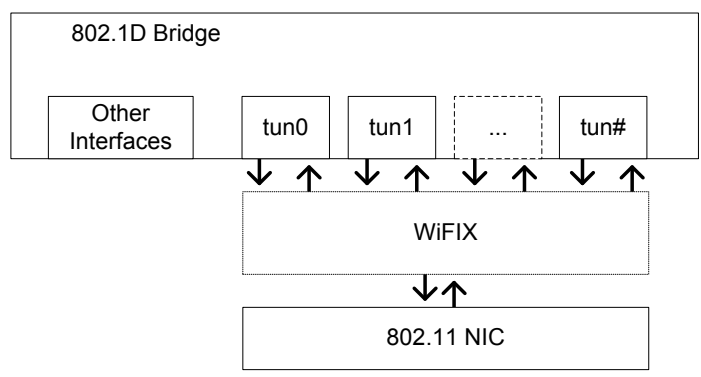

Figure 2. WiFIX interaction with its peer modules.

\section{PACE SCHEDULING MECHANISM}

\section{A. Rationale}

PACE has been designed with 802.11-based Stub WMN in mind and layer 2 forwarding among MAPs, and assumes that a logical tree topology is configured over the physical network using WiFIX; particular cases are a single chain or multiple chains terminating at a gateway. The rationale for a new scheme is the following:

- it makes sense using 802.11 as a basis due to its ubiquity, low cost and enhanced features of recent versions;

- basic CSMA/CA does not work in WMNs and the normal RTS/CTS handshake may even be counterproductive;

- CSMA/CA-related backoffs at each 802.11 link must be avoided, since they cause unfairness problems [20];

- solutions described in Section II to mitigate problems due to RTS/CTS and other effects are either too complex or their use has not been proved to apply to WMNs;

- centralised scheduling based on allocation of slots to non conflicting links, besides complex (except in simple cases), introduces non negligible control and synchronisation overhead and is not suitable for highly dynamic scenarios.

With CSMA/CA alone the nodes operate in an uncoordinated way and thus the rate of collisions increases with the offered load and the operation of the network becomes unstable beyond a critical point, on the onset of congestion. Due to traffic asymmetries it is difficult to predict this point and thus apply any form of source control to reach such objective. Even if this was feasible, the target throughput would be very small compared with the "ideal" capacity of the 
network due to the high number of collisions and retransmissions necessary to deliver the traffic generated with null or low losses. This claim is confirmed by ns-3 simulations, even in simple cases.

The main goal of the proposed mechanism is thus to provide coordinated access among nodes to prevent collisions, without requiring explicit synchronisation, as in slotted schemes, and allowing variable size packets. PACE is overlaid on CSMA/CA, which is still necessary to resolve possible collisions between control and data packets. For now, PACE addresses the problem without considering spatial reuse techniques; an extension that exploits spatial reuse has already been designed and will be the subject of a future paper.

\section{B. Description}

In a Stub WMN most of the traffic is exchanged with external networks through a gateway with low or null internal traffic between any pair of nodes. Thus, the gateway is the natural choice to act as the central controller, deciding the scheduling of the downstream traffic and the polling order and frequency for upstream traffic (the default being roll-call polling).

As mentioned, in each MAP, WiFIX runs on top of the 802.11 wireless card and processes all incoming/outgoing data frames. This allowed including PACE in WiFIX and keep backwards compatibility with the 802.11 MAC. PACE limits transmissions to a single WMN node at each time and ensures that each node has the opportunity to transmit a packet in each network-wide transmission round, allowing easy deployment of efficient and fair WMNs. It works as follows. The master MAP controls the access to the medium. When the network is bootstrapped the master MAP takes control and transmits a packet to a given destination MAP, which in turn will deliver the packet to the destination terminal attached to it; the packet received by the destination MAP is implicitly used as a permit to transmit a packet. Upon the packet coming from the destination MAP is received by the master MAP the same process is repeated with another destination MAP, until all the MAPs have had the opportunity to transmit one packet and the first destination MAP can be authorized to transmit again.

It is well known that control packets, albeit small, degrade performance, as will be confirmed in Section VI. Hence, when possible, a single interaction between the gateway and a polled node is used to exchange packets in both directions. In case no data is available, an explicit control packet is sent; otherwise, the polling signal from the gateway or the return control signal from the node are embedded in data packets.

During each interaction, packets have to hop through intermediate nodes that only act as relays. Sending a single packet is straightforward; for multiple packets the whole batch should be sent hop-by-hop to avoid intra-flow interference, thus requiring additional processing by the relay nodes. This is the reason why the default is sending one packet at most per poll. This defines a "cut-through"-like operation.

\section{Modifications to WiFIX}

The polling mechanism just described could not be directly implemented on the original WiFIX protocol due to its routing limitations. WiFIX does not create any route between nodes before data is injected into the network (learning bridge process). This means that when the polling mechanism was triggered the gateway (master MAP) could not send a polling request to a node at a topological distance higher than one hop. The WiFIX protocol was then upgraded to overcome this limitation. In the new version, the virtual tunnel between two mesh nodes (parent and child) is only created after the parent node receives an explicit unicast message sent by the child. Then, the parent forwards this message to the gateway to establish routes between all the intermediate nodes and that particular node, and to inform the gateway about the set of terminals that may be attached to that parent's child. With this method, the gateway knows the whole network topology, including the terminals attached to each MAP, and has the needed information to run the described PACE mechanism .

\section{NS-3 IMPLEMENTATION}

The implementation of the proposed protocol in network simulator 3 (ns-3 version 3.12.1) required four steps: (1) full implementation of the original WiFIX; (2) modifications to WiFIX to support PACE; (3) PACE implementation; (4) simulation of different scenarios based on CSMA/CA only and polling over CSMA/CA.

In step (1) none of the pre-established models included in ns-3 package installation were modified. Because WiFIX was designed to operate directly over the standard 802.11 MAC layer, for the sake of simplicity it was implemented in ns-3 at the Application layer using User Datagram Protocol (UDP) sockets to transfer packets.

The main modifications in step (2) were mentioned in Section IV; other modifications focused on improving the creation and refreshment of routes, avoiding the need for additional overhead messages.

In step (3) the polling scheme was integrated with WiFIX maintaining the data generation applications unaware of both mechanisms (WiFIX and polling).

In step (4) a set of scenarios was defined, firstly based on CSMA/CA and then polling over CSMA/CA, in order to assess the PACE gains in terms of stability, throughput and fairness, and the factors that may affect its performance due to the inherent control overheads.

\section{EVALUATION}

\section{A. Simulation Setup}

Although the limitations of CSMA/CA in multi-hop networks are well documented in the literature, pure CSMA/CA was simulated in the first place, not only to confirm its known problems but also to characterize and compare its performance with the polling scheme in similar scenarios and conditions (both network topology and traffic profiles). For the reasons discussed in Section III, RTS/CTS is disabled in all cases.

To put in evidence the serious limitations of CSMA/CA, even is simple cases, and to compare with polling, a layout of fives nodes (a gateway and four MAPs) arranged in a chain was initially considered, as represented in Fig. 3; in more complex topologies the performance of CSMA/CA would only worsen. 


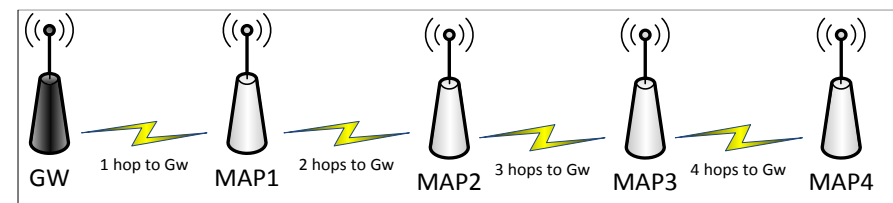

Figure 3. Illustration of the chain layout.

For the polling case, simulation results are also presented for a radial layout and a large number of nodes. The initial traffic scenarios and their purpose are described next.

\section{1) CSMA/CA only}

Case 1: only one MAP is active and generates traffic towards the gateway. This allows capturing the effect of intraflow interference and how performance degradation is affected by the number of hops. The offered load is increased so that it is possible to assess the maximum achievable throughput and the behaviour after the congestion point is reached.

Case 2: all MAPs are active and generate similar traffic towards the gateway. This also allows assessing the effect of inter-flow interference as well as performance degradation and unfairness with increasing rate of collisions and packet losses.

Bidirectional traffic in either case would only aggravate the overall behaviour.

\section{2) Polling over $C S M A / C A$}

Different cases are distinguished along three axes:

- only a single MAP is active (generates traffic) or all MAPs are active;

- only the active MAPs are polled (direct polling) or all MAPs are polled, whether active or not (full polling);

- traffic is unidirectional (from an active MAP towards the gateway) or bidirectional (both upstream and downstream).

Case 3: a single MAP is active, being the only one that is polled (direct polling) and traffic is bidirectional (the poll signal is embedded in downstream data). This allows assessing how the distance (hops) to the gateway affects performance, since the number of links affected by intra-flow interference, which must be avoided, is different for each active MAP.

Case 4 is similar to case 3 but with upstream traffic only; polling now requires a control packet and thus this also allows assessing the effect of not embedding the poll signal in data.

Cases 5 and 6 are similar to cases 3 and 4, respectively, but now all nodes are polled (full polling). This allows assessing the effect of polling inactive nodes (all but one).

Case 7: all four MAPs are active and traffic is bidirectional (the poll signal is embedded in data). This captures the effect of also preventing inter-flow interference (weighted over all nodes) and shows the fairness behaviour.

$\underline{\text { Case } 8}$ is similar to case 7 , but with upstream traffic only.

To enforce fairness (in the max-min sense) all MAPs send at most one data frame when polled. Similarly, a polling message carries at most one data frame for the polled MAP. In some cases polling frames do not carry data (upstream traffic only) and in other cases only one MAP is active (the others may be polled or not). In the full polling cases, each MAP is polled once in a cycle.

The following configuration settings were adopted.

\section{Simulator configuration}

RTS/CTS: disabled

Radio Configuration:

TxGain: $5 \mathrm{~dB}$ | RxGain: $5 \mathrm{~dB}$ | TxPowerEnd: $16 \mathrm{dBm}$ TxPowerstart: $16 \mathrm{dBm}$

EnergyDetectionThreshold: $-63 \mathrm{dBm}$

CcaModelThreshold: $-68 \mathrm{dBm}$

Propagation model: Friis Propagation Loss Model $900 \mathrm{MHz}$

Antenna height above soil: $1.5 \mathrm{~m}$

Error ratio model: Nist Error Rate Model

Communication standard: IEEE 802.11g

Mobility model: None

Number of runs per test: 300

Simulation time per run: $300 \mathrm{~s}$

\section{WiFIX configuration:}

Generating rate of topology refresh messages: $6 /$ minute Network warm-up time: $120 \mathrm{~s}$

\section{B. Simulation Results and Analysis}

In all simulations the size of data frames is 2000 bytes, which includes MAC header fields. Rate values are expressed in terms of goodput, as seen at the Application layer. All results are plotted with a $95 \%$ confidence interval.

Case $1-$ The worst situation occurs when only MAP4 is active, since it is at the highest distance from the gateway. Detailed graphical results are only presented for this MAP: the goodput and one-way delay in Fig. 4 and the packet loss ratio (at the Application layer) in Fig. 5.

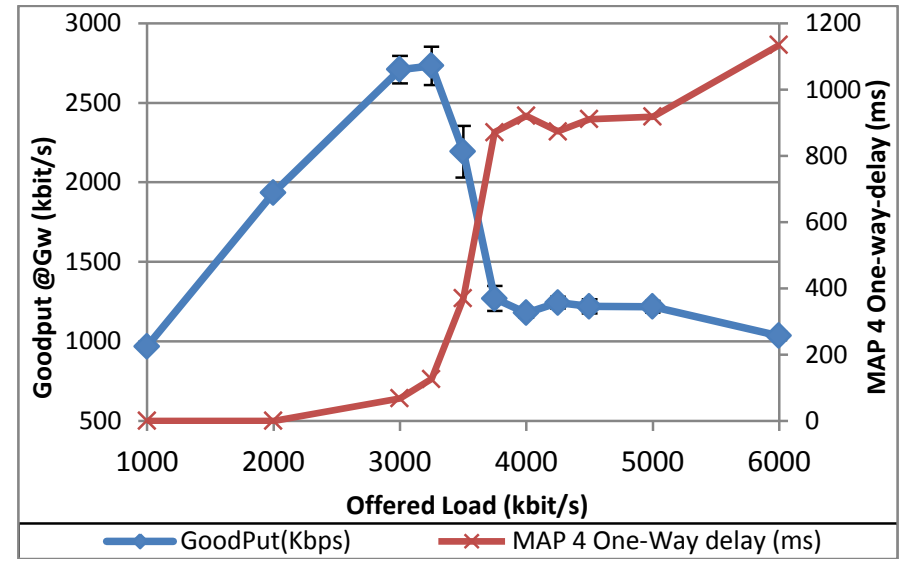

Figure 4. Goodput and one-way-delay of MAP 4 (CSMA/CA). 


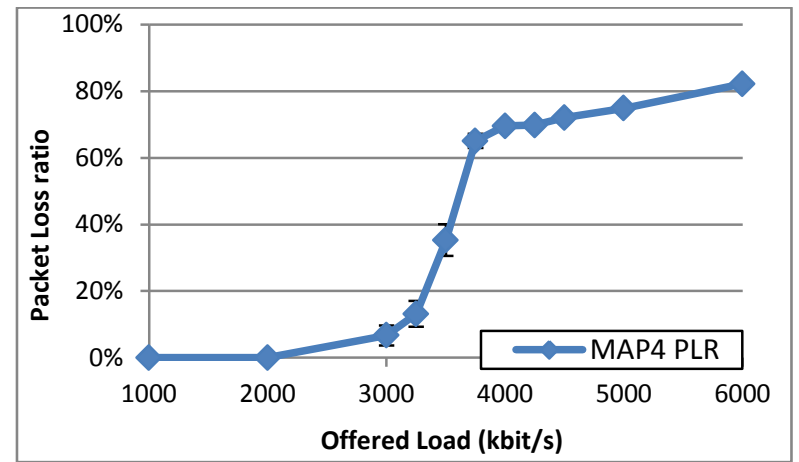

Figure 5. Packet loss ratio of MAP4 at Application layer (CSMA/CA)

Increasing the offered load, packet losses start to occur above $2 \mathrm{Mbit} / \mathrm{s}$; beyond this point the collision rate is such that some packets fail to be delivered. The maximum goodput is about $2.7 \mathrm{Mbit} / \mathrm{s}$ (only $5 \%$ of the nominal channel capacity of $54 \mathrm{Mbit} / \mathrm{s}$ ) for an offered load of $3.1 \mathrm{Mbit} / \mathrm{s}$ and for higher loads there is a sharp increase in the delay and packet loss ratio.

Simulations were also performed for the other MAPs. For MAP3 results are only slightly better (the maximum goodput is around $3.85 \mathrm{Mbit} / \mathrm{s}$ ), but there is still a strong effect due to interference of nodes not within carrier sensing range. If only MAP1 is active, and since the gateway does not generate traffic, there are no collisions and thus the maximum observed goodput (around $32 \mathrm{Mbit} / \mathrm{s}$ ) only depends on the overhead of the access protocol (including ACKs). This sets an absolute real upper bound (one hop without collisions) that may also be used as a reference for the polling cases.

Case 2 - All MAPs offer the same load, which is increased in steps of $50 \mathrm{kbit} / \mathrm{s}$, with smaller steps in the interval between 350 and $400 \mathrm{kbit} / \mathrm{s}$ for a more detailed analysis. Fig. 6 shows that beyond a critical point (around $300 \mathrm{kbit} / \mathrm{s}$ ) degradation is already obvious, with strong reduction of goodput of the nodes at higher distances from the gateway (MAP3 and MAP4).

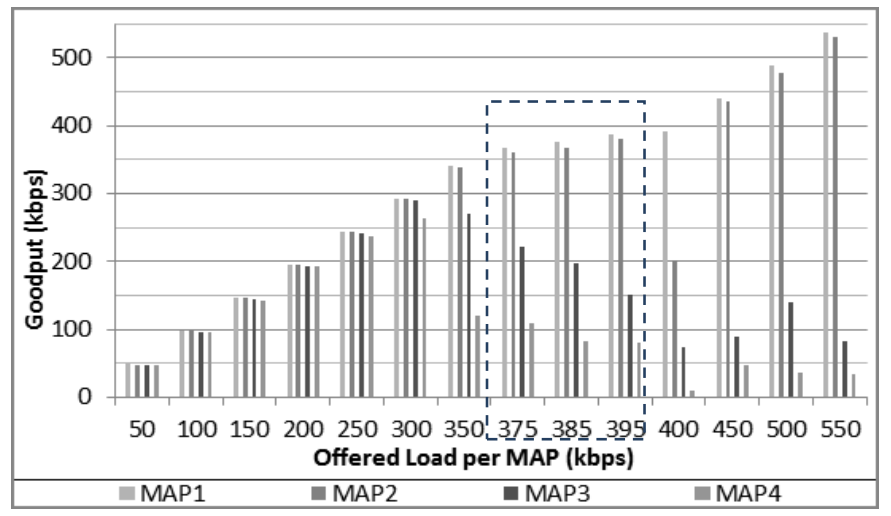

Figure 6. Goodput of each MAP when all are active (CSMA/CA).

At an offered load of $400 \mathrm{kbit} / \mathrm{s}$, MAP2 is penalised due to competition with MAP3 and MAP4, which still can get some traffic transmitted; at $450 \mathrm{kbit} / \mathrm{s}$ and beyond MAP2 recovers and shares the network capacity with MAP1 since the other two MAPs loose competition more frequently and may end up starving. For a total offered load of $2 \mathrm{Mbit} / \mathrm{s}$ (500 kbit/s per MAP), the total goodput is less than $1.2 \mathrm{Mbit} / \mathrm{s}$. The one way delay of each MAP shown in Fig. 7 further confirms how the distance to the gateway affects performance.

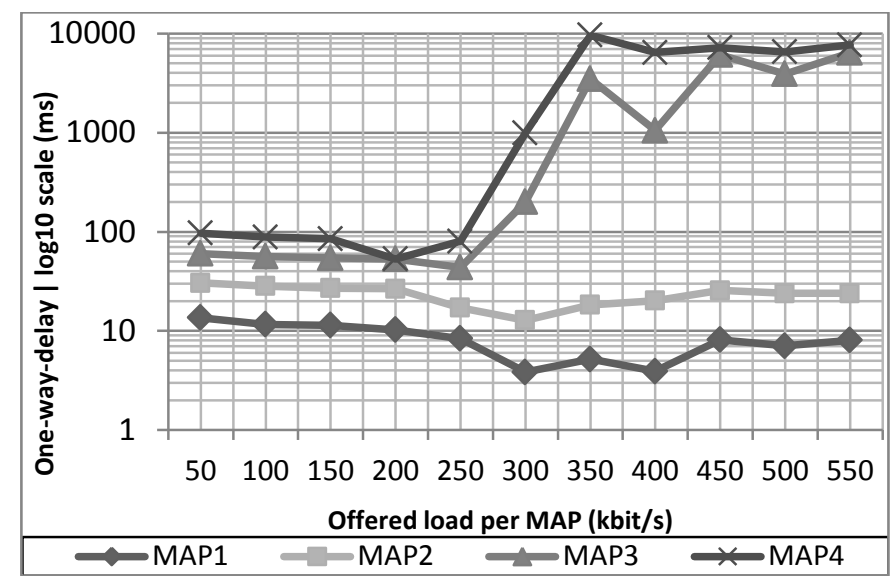

Figure 7. One-way-delay of each MAP when all are active (CSMA/CA).

The results confirm, in the simple case of a 4-hop chain, the serious performance degradation of multi-hop networks that only rely on CSMA/CA for controlling access to the medium.

For the same topology, the cases considered under polling put in evidence performance gains, which depend on traffic patterns and polling overhead, but also the stable and fair behaviour of the scheme, as demonstrated by simulation.

Fig. 8 shows the total goodput per MAP (adding both directions) for the polling cases. For cases 3 to 6 , these values correspond to independent simulations, since only one MAP is active at a time; thus, they coincide with the total goodput on the network. In cases 7 and 8 the total goodput on the network is the sum of the individual ones. Case 8 is not represented.

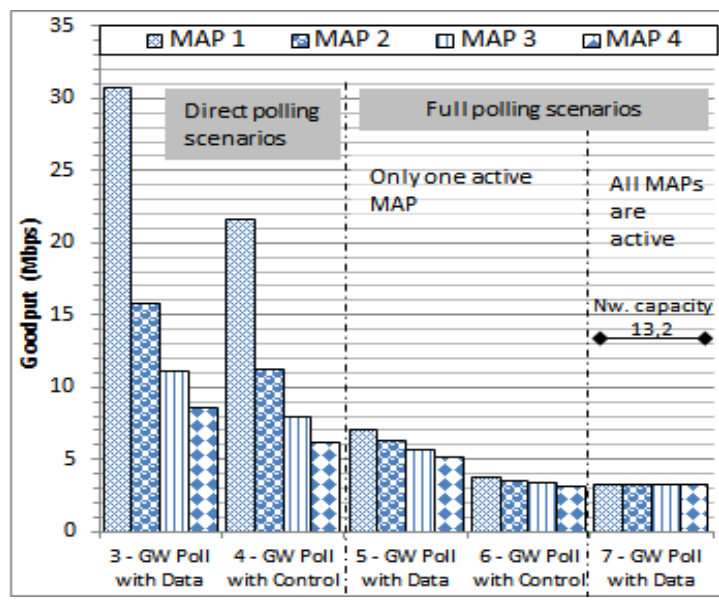

Figure 8. Goodput of each MAP in cases 3 to 7 (Polling).

Case 3 - Since in this case only one MAP is active, traffic is bidirectional, inactive MAPs are not polled and there are no collisions, maximum goodput is expected. In particular for MAP1, the highest goodput is achieved (full utilization of the useful channel capacity) and is similar to the reference value obtained in Case 1, but now with the channel capacity equally divided by both directions. For the other MAPs, goodput is roughly inversely proportional to the number of hops, as confirmed in Fig. 8; it is worth saying that MAP4 would have 
similar performance to MAP3 in case spatial reuse was exploited (spatial reuse is not possible in the other cases).

The results corresponding to Case 4 (unidirectional traffic) show a reduction of the total goodput due to the non negligible overhead of sending control packets for polling purposes; however, goodput in the upstream direction is higher than in the previous case, since bandwidth in the downlink direction is only used by control traffic.

Cases 5 and 6 differ from the previous ones due to the fact that inactive MAPs are also polled and thus there is further goodput reduction due to such additional overhead.

Cases 7 and 8 cover the situations where all MAPs share the network capacity, while avoiding both intra and inter-flow interference. In case 7 the total goodput (both directions) is 13.2 Mbit/s, that is 3.3 Mbit/s per MAP and $1.65 \mathrm{Mbit} / \mathrm{s}$ per MAP and direction. The total goodput is well in line with the fact that the average hop count is 2.5 , which means that a value in the order of 0.4 times the highest reference value would be expected. For unidirectional traffic (case 8), there is a further reduction, as explained for case 4: the total network goodput is 9.6 Mbit/s (2.4 Mbit/s per MAP), but the upstream values are higher than the corresponding ones in case 7.

The roundtrip times were also evaluated; for each MAP they are inversely proportional to the corresponding goodput.

Simulations for more complex topologies were carried out with a radial layout of nodes, with the gateway in the middle. Random topologies were created, an example of which is shown in Fig. 9.

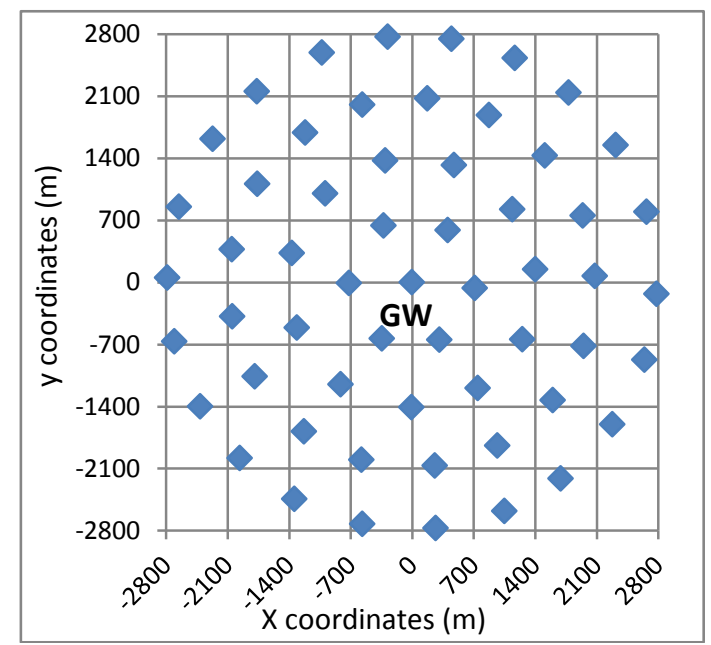

Figure 9. Radial layout illustration (example).

A number of simulations were performed in order to assess the behaviour of the scheme when varying the number of nodes and the average hop count. Fig. 10 shows an almost perfect match between the theoretical and the simulated values of the goodput as a function of the average hop count, for case 7 and a radial layout, assuming that both values coincide in the single hop case, which is used as a reference.

It is easy to derive that the theoretical maximum goodput (network capacity), under ideal conditions, with greedy sources but without spatial reuse, is inversely proportional to the average hop count (this was observed in the chain topology, and was mentioned when analysing case 7).

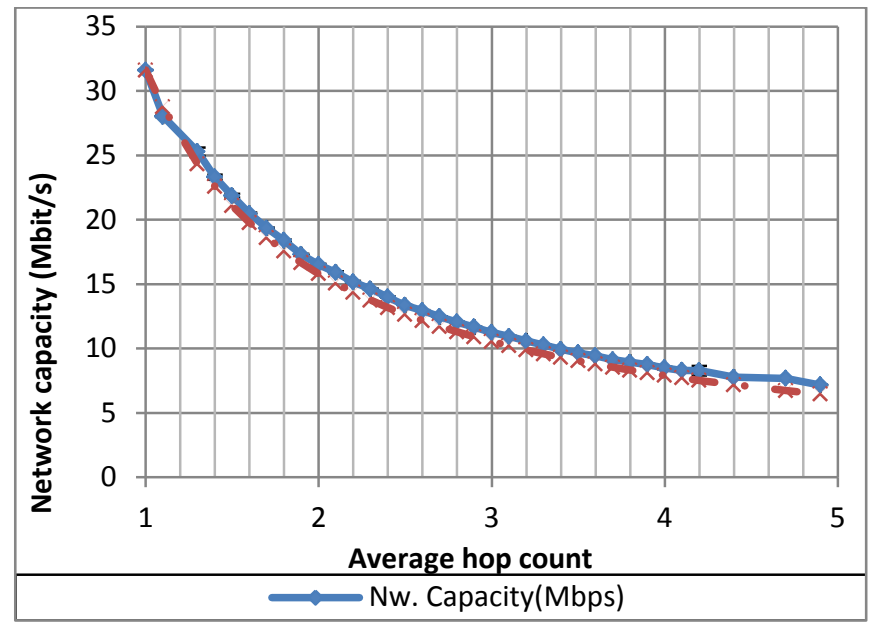

Figure 10. Network capacity obtained by simulation vs. the theoretical value for case 7 with radial layout.

The roundtrip time (referring to the transmission of 10 packets) and the average number of nodes as a function of the average hop count are represented in Fig. 11, again for case 7 and a radial layout.

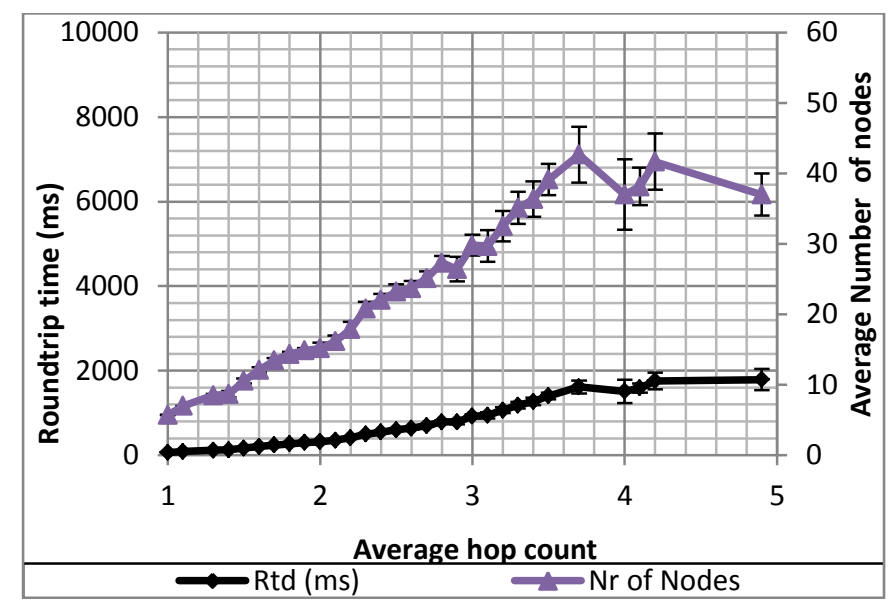

Figure 11. Average roundtrip time (10 packets) and average number of nodes for case 7 with radial layout.

\section{Discussion}

Based on the proposed concept and the simulation results we can confirm the added value of the proposed solution and identify its limitations and possible ways of overcoming them. Firstly, the proposed scheme is rather simple and backwards compatible with IEEE 802.11. Secondly, fairness is inherent to the method and confirmed by the results, when all MAPs are active. Together these features will contribute to: (1) further increase Stub WMN attractiveness by enabling Quality of Service that is independent of the hop count to the gateway, even supporting differentiated services, and (2) make the deployment of Stub WMNs easier and less expensive thanks to the use of commodity 802.11 hardware.

With PACE, performance degrades as the average number of hops increases. However, the degradation is sharper for average hop counts up to three and this is inevitable due to interference of neighbour links. For larger values of the average hop count the degradation is smoother and may be 
mitigated by means of spatial reuse, which can also be applied to nodes closer to the gateway provided that there is enough spatial diversity (from this point of view the most unfavourable topologies are chains or trees with first tier nodes not sparsely distributed in space). On the negative side there is a wastage of resources when it is not possible to embed polling signals in data frames; sending a frame, even short, has a high price in CSMA/CA-oriented networks and thus such overhead is non negligible. Polling nodes without traffic also has a similar price, since a control frame is also required. The worst situation occurs when for a given node there is no traffic in both directions. In other words, the need to send a high number of control frames per cycle contributes to further degrading the performance; this may be mitigated by using intelligent variants of polling, other than the roll-call currently used. In any case, active nodes still benefit from a larger capacity than when having to share resources with additional ones.

The proposed scheme is suitable for networks with a moderate average hop count. For larger networks it may be improved with spatial reuse techniques. A novel technique has been designed and is being evaluated; it will be the subject of a future paper. On the other hand, PACE is mainly suitable for near-saturation or saturated WMNs; for non-saturated WMNs the traditional CSMA/CA method may achieve better performance than PACE. As future work, we plan to develop a new dynamic mechanism that enables or disables PACE according to the offered load.

\section{CONCLUSION}

IEEE 802.11-based WMNs are a cost-effective and flexible solution to extend wired network infrastructures, but suffer from two major problems: inefficiency and unfairness. To solve these problems we proposed PACE, a simple multi-hop scheduling mechanism for Stub WMNs overlaid on the IEEE 802.11 MAC. By means of simulations we proved that PACE can achieve optimal network capacity utilization and greatly outperform state of the art CSMA/CA-based solutions in terms of goodput, delay, and fairness. We plan to enhance PACE with additional QoS mechanisms, develop a new dynamic mechanism that enables PACE according to the offered load, and employ spatial reuse techniques that can make PACE applicable to large 802.11-based single-radioWMNs.

\section{ACKNOWLEDGMENT}

This work was financed by the European Commission under Grant Agreement No. FP7-288535 "CONFINE": Open Call 1, by ERDF - European Regional Development Fund through the COMPETE Programme (operational programme for competitiveness) and by National Funds through FCT Fundação para a Ciência e a Tecnologia (Portuguese Foundation for Science and Technology) within project «FCOMP - 01-0124-FEDER-022701».

\section{REFERENCES}

[1] R. Campos, R. Duarte, F. Sousa, M. Ricardo, J. Ruela, "Network Infrastructure Extension Using 802.1D-based Wireless Mesh Networks", Wireless Communications and Mobile Computing, vol. 11, Issue 1, January 2011, pp. 67-89.

[2] S. Ray, D. Starobinski, "On False Blocking in RTS/CTS-Based Multihop Wireless Networks", IEEE Transaction on Vehicular Technology, Vol. 56, No. 2, March 2007, pp. 849-862.
[3] B. Alawieh, Y. Zhang, C. Assi, H. Mouftah, "Improving Spatial Ruse in Multihop Wireless Networks", IEEE Communications Surveys \& Tutorials, Vol. 11, No. 3, Third Quarter 2009, pp. 71-91.

[4] K. Kosek-Szott, "A survey of MAC layer solutions to the hidden node problem in ad-hoc networks", Ad Hoc Networks, Vol. 10, No. 3, May 2012, pp. 635-660.

[5] R. Campos, "Joint Path and Address Auto-configuration: an Approach to Multi-technology Personal Area Networks and 802.11-based Stub Wireless Mesh Networks", PhD thesis, Univ. Porto, Portugal, July 2011.

[6] L.B. Jiang, S.C. Liew, "Improving Throughput and Fairness by Reducing Exposed and Hidden Nodes in 802.11 Networks", IEEE Transactions on Mobile Computing, Vol. 7, No. 1, January 2008, pp. 34 49.

[7] K. Xu, M. Gerla, S. Bae, "How Effective is the IEEE 802.11 RTS/CTS Handshake in Ad Hoc Networks", IEEE Globecom 2002, November 2002, Taiwan, Vol. 1, pp. 72-76.

[8] L.B. Jiang, S.C. Liew, "Hidden-node Removal and Its Application in Cellular WiFi Networks", IEEE Transactions on Vehicular Technology, Vol. 56, No. 5, September 2007, pp. 2641-2654.

[9] K. Jamshaid, P. Ward, "Gateway-Assisted Max-Min Rate allocation for Wireless Mesh Networks", MSWiM'09 - ACM/IEEE International Conference on Modelling, Analysis and Simulation of Wireless and Mobile Systems, October 2009, Tenerife, Spain.

[10] G. Sharma, N. Shroff, R. Mazumdar, "Joint Congestion Control and Distributed Scheduling for Throughput Guarantees in Wireless Networks", INFOCOM 2007 - 26th Annual IEEE Conference on Computer Communications, May 2007, Anchorage, USA.

[11] S. Razak, V. Kolar, N. Abu-Ghazaleh, K. Harras, "How do Wireless Chains Behave? The Impact of MAC Interactions", MSWiM'09 ACM/IEEE International Conference on Modelling, Analysis and Simulation of Wireless and Mobile Systems, October 2009, Tenerife, Spain, pp. 212-220.

[12] J. Li, C. Blake, D. De Couto, H. Lee, R. Morris, "Capacity of Ad Hoc Wireless Networks", ACM SIGMOBILE - 7th International Conference on Mobile Computing and Networks, July 2001, Rome, Italy, pp. 61-69.

[13] V. Gambiroza, B. Sadeghi, E. Knightly, "End-to-End Performance and Fairness in Multihop Wireless Backhaul Networks", MobiCom'04 10th Annual International Conference on Mobile Computing and Networking, September 2004, Philadelphia, USA, pp. 287-301.

[14] T. Nandagopal, T. Kim, X. Gao, V. Bharghavan, "Achieving MAC Layer Fairness in Wireless Packet Networks", MobiCom'00 - 6th Annual International Conference on Mobile Computing and Networking, August 2000, Boston, USA.

[15] L. Tassiulas, A. Ephremides, "Stability properties of constrained queueing systems and scheduling policies for maximum throughput in multihop radio networks", IEEE Transactions on Automatic Control, Vol. 37, No. 12, December 1992, pp. 1936-1948.

[16] G. Gupta, N. Shroff, "Practical scheduling schemes with throughput guarantees for multi-hop wireless networks", Computer Networks, Vol. 54, No. 5, April 2010, pp. 766-780.

[17] A. Warrier, S. Janakiraman, S. Ha, I. Rhee, "DiffQ: Practical Differential Backlog Congestion Control for Wireless Networks", INFOCOM 2009 - 28th Annual IEEE Conference on Computer Communications, April 2009, Rio de Janeiro, Brazil, pp. 262-270.

[18] U. Akyol, M. Andrews, P. Gupta, J. Hobby, I. Saniee, A. Stolyar, "Joint Scheduling and Congestion Control in Mobile Ad-Hoc Networks", INFOCOM 2008 - 27th Annual IEEE Conference on Computer Communications, April 2008, Phoenix, USA, pp. 619-627.

[19] G. Sharma, R. Mazumdar, N. Shroff, "On the Complexity of Scheduling in Wireless Networks", MobiCom'06 - 12th Annual International Conference on Mobile Computing and Networking, New York, USA, pp. 227-238.

[20] S. Chakraborty, S. Nandi, "MAC Layer Fairness in IEEE 802.11 DCF Based Wireless Mesh Networks", International Conference on Computing, Networking and Communications, February 2012, Maui, Hawaii, USA. 